\title{
Efectos combinados del fuego y el ganado en matorrales y bosques del noroeste patagónico
}

\author{
Melisa Blackhall ${ }^{1, \bowtie}$; Estela Raffaele ${ }^{1} \&$ Thomas T. Veblen ${ }^{2}$ \\ 1. Laboratorio Ecotono, Universidad Nacional del Comahue, INIBIOMA-CONICET, Bariloche, Argentina. 2. Department of \\ Geography, University of Colorado, USA.
}

\begin{abstract}
REsumen. En el noroeste patagónico, dos de los disturbios de mayor influencia sobre los paisajes y comunidades son el fuego y la herbivoría de ungulados introducidos. Existe una larga historia regional del fuego, pero la reiteración de incendios seguidos de intenso pastoreo constituye un nuevo tipo de régimen de disturbio en este paisaje. Nuestro objetivo fue analizar distintas condiciones micro-ambientales y parámetros de la vegetación en sitios afectados por la combinación de ambos disturbios. Dentro del Parque Nacional Nahuel Huapi, estudiamos 17 sitios bajo la influencia del fuego y el ganado vacuno: sitios no quemados ( $>50$ años) o recientemente quemados ( $<15$ años); y sin ganado o con herbivoría intensa. Encontramos un efecto contundente del tiempo transcurrido desde el último incendio y en menor medida del ganado. Ambos disturbios combinados sólo mostraron un efecto sinérgico sobre la humedad (durante las horas de mayor temperatura) y sobre la disponibilidad de luz, aumentando las condiciones adversas en los sitios recientemente quemados y con ganado. La cobertura total de especies leñosas y semi-leñosas también disminuyó en los sitios afectados por ambos disturbios. Maytenus chubutensis y Rosa rubiginosa caracterizaron los sitios no quemados y las especies representativas de los sitios con y sin ganado fueron Berberis darwinii y Ribes magellanicum, respectivamente. A través de diferentes mecanismos, el fuego y el ganado modifican las características de las comunidades. En algunos casos, estos cambios pueden ser mediados por el efecto conjunto y sinérgico de ambos disturbios produciendo condiciones ambientales más xéricas que aceleran la degradación de las tierras. El conocimiento de las especies indicadoras y la caracterización del micro-sitio en donde crecen brindan información básica para el desarrollo de pautas de restauración, conservación y manejo sustentable de bosques y matorrales patagónicos.
\end{abstract}

[Palabras clave: herbivoría, Nothofagus spp., regeneración post-fuego, condiciones micro-ambientales, especies indicadoras]

\begin{abstract}
Aвstract. Combined effects of fire and cattle in shrublands and forests of northwest Patagonia: In northwest Patagonia, the two most pervasive disturbances at the landscape and community levels are fire and herbivory by introduced ungulates. There is a long history of fire in the region, but repeated fires followed by heavy browsing constitute a new type of disturbance regime in these landscapes. Our objective was to analyze different micro-environmental variables and plant community parameters at sites affected by the combination of both disturbances. We studied 17 sites within Nahuel Huapi National Park, classified according to fire and herbivore history: not burned ( $>50$ years) or recently burned ( $<15$ years); and free of livestock or alternatively under heavy browsing. We found a strong effect of time since fire and a slighter effect of cattle. The combination of both disturbances only had a synergistic effect on humidity (during hours of higher temperature occurrence) and on solar radiation, and consequently an increase in adverse environmental conditions was observed at recently burned sites and with cattle present. Additionally, total cover of woody and semi-woody species decreased at sites affected by both disturbances. Maytenus chubutensis and Rosa rubiginosa characterized unburned sites; and indicator species at sites without cattle and with cattle were Ribes magellanicum and Berberis darwinii, respectively. Through different mechanisms, fire and cattle modify community characteristics, and when both disturbances co-occur, in some cases they can synergistically affect the plant communities, exacerbating xeric conditions which may accelerate land degradation. By increasing knowledge of indicator species and their associated site conditions, we can provide basic information for restoration guidelines, conservation, and sustainable management of Patagonian forests and shrublands.
\end{abstract}

[Keywords: herbivory, Nothofagus spp., post-fire regeneration, microsite conditions, indicator species]

\section{INTRODUCCIÓN}

Uno de los grandes desafíos pendientes de la ecología contemporánea sigue siendo entender y dilucidar las interacciones entre múltiples factores, incluidos los disturbios de diversos orígenes y a diferentes escalas espacio-temporales (Darling \& Côté 2008;

Editora asociada: Ana Cingolani

$\bowtie$ meliblackhall@gmail.com
Turner 2010). Recientemente, dentro de las prioridades enumeradas por Turner (2010) para el estudio de la ecología de disturbios destacan el elucidar y esclarecer dónde, cuándo y cómo la combinación de disturbios podría producir efectos sinérgicos o antagónicos, y de esta manera amplificar o atenuar el efecto total sobre los diferentes sistemas. Resulta 
difícil predecir las trayectorias sucesionales de un sistema cuando es afectado por disturbios combinados, ya sean naturales o antropogénicos (Paine et al. 1998), siendo además, los cambios micro-ambientales y las respuestas de las comunidades, en la mayoría de los casos, desconocidas. El fuego y la herbivoría son considerados los mayores consumidores de vegetación a nivel global (Bond \& van Wilgen 1996). El efecto directo de ambos disturbios es el consumo parcial o total de la vegetación: el fuego actúa como factor abiótico (consumiendo compuestos orgánicos complejos y convirtiéndolos en productos de combustión), mientras que la herbivoría a través de la remoción directa de biomasa actúa como factor biótico (Bond 2005). Sin embargo, pocos trabajos se han focalizado en el estudio combinado de ambos disturbios como modeladores de la vegetación (Bond 2005; e.g. Collins 1987; Tercero-Bucardo et al 2007; Fuhlendorf et al. 2009; Kirkpatrick et al. 2011; Raffaele et al 2011; Blackhall 2012).

Particularmente en el noroeste patagónico, dos de los disturbios que influyen en mayor medida y a diferentes escalas sobre el paisaje y las comunidades son el fuego y la herbivoría de mamíferos introducidos (Kitzberger \& Veblen 1999; Raffaele et al. 2011; Veblen et al. 2011). En esta región, y a lo largo del gradiente ambiental estepa-bosque lluvioso (este-oeste), los incendios naturales y antropogénicos han sido factores claves en la formación de los paisajes que hoy observamos (Veblen et al. 2003). En el noroeste de la Patagonia se ha registrado a escala de paisaje que el fuego afecta de diferente manera los distintos tipos de vegetación (Mermoz et al. 2005). Los matorrales xerofíticos dominados por especies como Nothofagus antarctica y Chusquea culeou (la nomenclatura de las especies sigue a Correa (1969-1997)), entre otras, son en general más afectados por eventos de fuego que los bosques mesofíticos adyacentes (Mermoz et al. 2005). Por ejemplo, los bosques de Nothofagus pumilio son menos susceptibles al fuego y a su propagación, a menos que las condiciones de sequía sean extremas (Mermoz et al. 2005).

Además de los efectos a escala de paisaje, también se ha encontrado en la región un importante efecto del fuego como modificador de micro-sitios, incluyendo cambios en la disponibilidad de nutrientes, radiación solar, temperaturas y humedades relativas (e.g. Alauzis et al. 2004; Kitzberger et al. 2005; Paritsis et al. 2006). Estos cambios pueden ser conducidos por efectos directos del fuego (e.g. incremento de conductividad eléctrica y $\mathrm{pH}$ del suelo, disminución de $\mathrm{N}$ total en suelo; Alauzis et al. 2004), o por efectos indirectos mediados a través del consumo total o parcial de la vegetación (e.g. mayor radiación y temperaturas, y menor humedad relativa por ausencia de vegetación; Kitzberger et al. 2005; Paritsis et al. 2006).

Superpuesto al efecto del fuego como modelador ambiental regional y consumidor abiótico de la vegetación, los grandes herbívoros también juegan un rol importante actuando como consumidores bióticos, especialmente a partir de la introducción de ungulados exóticos como ganado (principalmente vacuno) y cérvidos (Merino et al. 2009). En 1999 se estimó que un 56 \% de la superficie terrestre abarcada por el Parque Nacional Nahuel Huapi (706000 ha aprox.) era afectada por diferentes intensidades de ganado vacuno (Lauría Sorge \& Romero 1999). En una amplia variedad de tipos de bosques y matorrales del noroeste patagónico se han estudiado los efectos de los herbívoros exóticos sobre la regeneración post-fuego (Kitzberger et al. 2005; Tercero-Bucardo et al. 2007; Blackhall et al. 2008), y se ha demostrado que la herbivoría intensa cambia la estructura de las comunidades (Raffaele et al. 2011), y modifica las características intrínsecas de las especies relacionadas con su inflamabilidad y combustible, potenciando así el riesgo de incendio (Blackhall et al. 2012; Blackhall et al. 2015).

Existen numerosas evidencias de una larga historia de fuego en la región (Kitzberger \& Veblen 1999), pero frecuentemente la reiteración de incendios seguidos de intenso pastoreo por ganado exótico constituye un nuevo tipo de régimen de disturbio en este paisaje (Blackhall et al. 2008; Raffaele et al. 2011; Veblen et al. 2011). El objetivo de este trabajo fue comparar variables microambientales (e.g. temperaturas, amplitud térmica, humedad relativa, disponibilidad de luz) y parámetros de la vegetación (e.g. cobertura, riqueza, especies indicadoras) entre sitios afectados de forma diferente por la combinación del fuego (tiempo desde el último incendio) y la herbivoría de ganado vacuno. Esperamos encontrar un efecto combinado del tiempo transcurrido desde el último incendio y del ganado sobre las variables micro-ambientales y los diferentes parámetros que describen las comunidades vegetales, observando particularmente un efecto sinérgico en los sitios recientemente quemados con ganado. 


\section{MÉTOdos}

\section{Área de estudio}

Este estudio fue realizado en el Parque Nacional Nahuel Huapi, Provincia de Río Negro, Argentina. En esta latitud, la región está subdividida fisiográficamente de oeste a este en la cordillera de los Andes, la pre-cordillera, y la estepa patagónica. El clima de la región es del tipo Mediterráneo, con precipitaciones en otoño-invierno y un período seco y caluroso en primavera-verano. Los suelos son de origen volcánico. En la zona del lago Nahuel Huapi la temperatura máxima media del mes más cálido es de $21.7^{\circ} \mathrm{C}$ (febrero), la mínima media del mes más frío es de $-0.5^{\circ} \mathrm{C}$ (julio), y la media general anual es de $8.9^{\circ} \mathrm{C}$ (promedios 1998-2012; Autoridad Interjurisdiccional de las Cuencas de los Ríos Limay, Neuquén y Negro (AIC)). La precipitación media anual decrece abruptamente de oeste a este, oscilando entre $4000 \mathrm{~mm}$ en la cordillera de los Andes y menos de $500 \mathrm{~mm}$ en la estepa patagónica (Barros et al. 1983). A altitudes medias (800-1100 m s.n.m.), los bosques perennifolios son dominados por la conífera xerófila Austrocedrus chilensis y/o la angiosperma mesofítica Nothofagus dombeyi; a mayores altitudes $(\approx 1000-1500$ m s.n.m.) los bosques subalpinos son mono-específicos y están dominados por la especie decidua y mesofítica N. pumilio (Mermoz \& Martin 2005). Las tres especies se reproducen obligadamente por semilla y no poseen la capacidad de rebrotar luego de un incendio (Veblen et al. 2003). En sitios relativamente más secos y próximos a los bosques maduros se observan matorrales altos (2-5 m aprox.), que típicamente reemplazan a los bosques durante la sucesión temprana post-fuego. Estos matorrales xerofíticos son dominados en general por la especie $N$. antarctica (que crece en forma de árbol o arbusto según condiciones del sitio y es una especie decidua; Donoso et al. 2006), la bambusoidea Chusquea culeou y un gran número de especies de pequeños árboles y arbustos altos. La mayoría de estas especies rebrotan vigorosamente luego de un incendio (Veblen et al. 2003). En estos hábitats algunas especies que comúnmente acompañan a $N$. antarctica son Lomatia hirsuta, Schinus patagonicus, Maytenus boaria, Berberis spp., y Diostea juncea (Mermoz \& Martin 2005).

Se estudiaron diferentes sitios en bosques y matorrales según los siguientes dos criterios: a) tiempo transcurrido desde el último incendio: recientemente quemado (entre 10 a 15 años desde el último evento de fuego); ó alternativamente no quemado al menos en los últimos 50 años; y b) presencia de ganado vacuno: libre de ganado; ó alternativamente con presencia histórica y actual de ganado durante al menos los últimos 25 años. De esta forma, se seleccionaron ocho sitios no quemados: cuatro sin ganado (Q- G-) y cuatro con presencia de ganado vacuno $(\mathrm{Q}-\mathrm{G}+)$; y nueve sitios quemados recientemente: cinco sin ganado $(\mathrm{Q}+\mathrm{G}-)$, y cuatro con presencia de ganado vacuno $(\mathrm{Q}+\mathrm{G}+)$ (Tabla 1). Todos los sitios se encuentran en la zona mésica del gradiente (entre 1000 y $1700 \mathrm{~mm}$ de precipitación media anual). Los sitios clasificados como recientemente quemados fueron afectados por incendios de origen antrópico ocurridos luego de sequías extremas (1996: aprox. 600 has; 1999: aprox. 4500 has; Administración de Parques Nacionales 1999; Tabla 1). Estos sitios recientemente quemados se caracterizaron por la falta del dosel arbóreo, ya que se encontraban en un estadio de sucesión temprana post-fuego. Los sitios con ganado vacuno se caracterizaron por registrar una larga e intensa historia de pastoreo, al menos durante los últimos 25 años (Lauría Sorge \& Romero 1999). Mediante observaciones de campo (e.g. heces, huellas, raspado) y averiguaciones en instituciones idóneas (e.g. Parques Nacionales, Guardaparques) se constató para cada sitio la ausencia de otros grandes herbívoros en los sitios de muestreo, en particular de cérvidos introducidos.

Tabla 1. Características de los sitios; F: afectado o no por fuego reciente (no quemado (Q-), quemado $(\mathrm{Q}+)$ ); G: presencia de ganado vacuno (sin ganado (G-), con ganado vacuno $(\mathrm{G}+)$ ); AI: año del último incendio: $>50=$ último incendio registrado hace más de 50 años); PG: presión de ganado; Alt: altitud (m s.n.m); PMA: precipitación media anual (mm/año); y especies arbóreas dominantes en cada tipo de bosque (actuales o previas al incendio reciente). Fuentes: Administración de Parques Nacionales 1999; Lauría Sorge y Romero 1999; base WORLDCLIM: Hijmans et al. 2005; registros a campo y datos no publicados.

Table 1. Characteristics of the studied sites; F: affected or not by recent fire (non-burned (Q-), burned $(\mathrm{Q}+)$ ); $\mathrm{G}$ : presence of cattle (without cattle $(\mathrm{G}-)$, with cattle $(\mathrm{G}+)$ ); AI: year of occurrence of last fire: $>50$ years $=$ last fire occurred more than 50 years ago); PG: cattle pressure; Alt: altitude (m s.n.m.); PMA: mean annual precipitation (mm/year); and dominant tree species for each type of forest (present or previously to fire). References: details in the Spanish caption.

\begin{tabular}{|c|c|c|c|c|c|c|}
\hline$\underline{\text { Sitio }}$ & F G & AI & PG & Alt & PMA & Tipo de Bosque \\
\hline 1 & Q- G- & $>50$ & Nula & 790 & 1108 & N. dombeyi-A. chilensis \\
\hline 2 & Q- G- & $>50$ & Nula & 849 & 1064 & N. dombeyi - A. chilensis \\
\hline 3 & Q- G- & $>50$ & Nula & 851 & 1093 & N. dombeyi - N. antarctica \\
\hline 4 & Q- G- & $>50$ & Nula & 826 & 1059 & N. dombeyi - A. chilensis \\
\hline 5 & Q- G+ & $>50$ & Intensa & 967 & 1235 & N. dombeyi - N. antarctica \\
\hline 6 & Q- G+ & $>50$ & Intensa & 821 & 1363 & N. dombeyi - N. antarctica \\
\hline 7 & Q- G+ & $>50$ & Intensa & 800 & 1710 & N. antarctica \\
\hline 8 & Q- G+ & $>50$ & Intensa & 766 & 1732 & N. dombeyi - A. chilensis \\
\hline 9 & $\mathrm{Q}+\mathrm{G}-$ & 1996 & Nula & 859 & 1079 & N. dombeyi - A. chilensis \\
\hline 10 & $\mathrm{Q}+\mathrm{G}^{-}$ & 1996 & Nula & 866 & 1039 & A. chilensis \\
\hline 11 & $\mathrm{Q}+\mathrm{G}-$ & 1999 & Nula & 1103 & 1123 & N. pumilio \\
\hline 12 & $\mathrm{Q}+\mathrm{G}-$ & 1999 & Nula & 1074 & 1123 & N. antarctica \\
\hline 13 & $\mathrm{Q}+\mathrm{G}-$ & 1999 & Nula & 849 & 1364 & N. dombeyi - A. chilensis \\
\hline 14 & $\mathrm{Q}+\mathrm{G}+$ & 1999 & Intensa & 1103 & 1123 & N. pumilio \\
\hline 15 & $\mathrm{Q}+\mathrm{G}+$ & 1999 & Intensa & 1074 & 1123 & N. antarctica \\
\hline 16 & $\mathrm{Q}+\mathrm{G}+$ & 1999 & Intensa & 827 & 1401 & N. dombeyi - N. antarctica \\
\hline 17 & $\mathrm{Q}+\mathrm{G}+$ & 1999 & Intensa & 897 & 1347 & N. antarctica \\
\hline
\end{tabular}

\section{Mediciones micro-ambientales}

En cada sitio se colocó un sensor de temperatura y humedad HOBO ${ }^{\circledR}$ TMC6-HA, conectado a un colector de datos $\mathrm{HOBO}{ }^{\circledR} \mathrm{H} 8$, a una altura de 120 $\mathrm{cm}$ sobre la superficie del suelo, con orientación 
norte y al resguardo de la luz y precipitaciones directas. El periodo de mediciones fue desde diciembre del 2009 a fines de marzo del 2010. Las lecturas de temperatura media $\left({ }^{\circ} \mathrm{C}\right)$ y humedad relativa (HR \%) del aire fueron tomadas cada día a intervalos de cuatro horas (seis mediciones por día, a las 0:00, 4:00, 8:00, 12:00, 16:00, y 20:00h). Además para cada sitio se calculó la amplitud térmica diaria media. Todos los registros (temperatura media y humedad relativa para cada horario de medición, y amplitud térmica diaria media) se promediaron por sitios para toda la temporada estival de mediciones, que corresponde con la temporada de crecimiento vegetal y con la temporada de ocurrencia de incendios.

La disponibilidad de luz solar se estimó mediante fotos hemisféricas tomadas en cada sitio (Rich 1990), utilizando una cámara digital (CoolPix 995, Nikon) apuntando al cenit y utilizando una lente convexa calibrada tipo ojo de pez (FCE8, Nikon) con un campo de $180^{\circ}$ Las fotografías se tomaron a 100 $\mathrm{cm}$ de altura y niveladas horizontalmente; luego se analizaron digitalmente (WinSCANOPY TM). Se tomaron al menos 6 fotos por sitio, en una misma fecha y durante enero y febrero. Los parámetros estimados para cada fotografía fueron: factor de sitio directo (FSD) y factor de sitio indirecto (FSI), donde FSD es la proporción de luz solar directa y FSI es la proporción de luz solar difusa debajo del dosel en relación a la recibida por encima del dosel (Rich 1990). WinSCANOPY calcula un valor medio predicho a partir del modelado de la radiación para toda la temporada de crecimiento (i.e. NoviembreAbril). Los valores por cada sitio se promediaron.

\section{Mediciones de vegetación}

Se realizaron muestreos a lo largo de cinco transectas de $50 \mathrm{~m}$ por sitio. Sobre dos puntos a lo largo de cada transecta se delinearon parcelas de 16 $\mathrm{m}^{2}$ y en ellas se registró la riqueza total de especies leñosas y semi-leñosas (e.g. C.culeou y las trepadoras Vicia nigricans y Mutisia spp.) en el estrato bajo, y se estimó visualmente la cobertura de cada una utilizando una escala de $0-100 \%$ para cada especie. En los sitios no quemados se registró la cobertura por debajo del dosel arbóreo, mientras que en los sitios quemados se registró la de toda la vegetación del único estrato presente. Además, en cada parcela de los sitios no quemados recientemente se registró la cobertura del dosel arbóreo (> $6 \mathrm{~m}$ de altura), identificando cada especie arbórea. A partir de estos datos se calculó para cada parcela la cobertura absoluta de cada especie, la cobertura total (suma de coberturas de todas las especies en cada parcela), la cobertura total del dosel arbóreo (para sitios no quemados) y el número total de especies. Todos los valores obtenidos por parcela se promediaron para cada sitio.

\section{Herbivoría}

Para corroborar que la historia de uso elegida para cada sitio fuera la correcta, sobre cada transecta delineada para las mediciones de vegetación se muestrearon además cinco parcelas de $2 \mathrm{~m}^{2}$ asignando en ellas tasas de ramoneo a cada especie leñosa y semi-leñosa presente. Para cada especie registrada se estimó un índice de ramoneo, adaptando la metodología usada en Veblen et al. (1992). Se asignaron diferentes tasas de ramoneo (TR), basadas en observaciones de campo y acordadas por dos observadores: 0 (nulo: $0 \%$ de ramas ramoneadas), 1 (leve: $0-25 \%$ de ramas comidas), 2 (moderado: $25-50 \%$ de ramas comidas) y 3 (intenso: más del 50\% de ramas comidas) (adaptado de Veblen et al. 1992; Blackhall et al. 2008).

\section{Análisis de datos}

Para analizar el efecto del fuego y la presión de ganado sobre la temperatura media y la humedad relativa por horario, la amplitud térmica diaria, la disponibilidad de luz (FSD y FSI), la cobertura total promedio y el número total promedio de especies se realizaron ANOVAs factoriales $2 \times 2$ (fuego: no quemado y recientemente quemado; ganado: ausencia y presencia; Quinn \& Keough 2002). Mediante un ANOVA de una vía se comparó la cobertura (\%) del dosel arbóreo de los sitios no quemados, para corroborar posibles diferencias debidas al efecto de la presencia de herbívoros (sitios Q- G- vs. sitios Q- G+). La normalidad de residuales fue evaluada mediante pruebas de Shapiro-Wilk y la homogeneidad de variancias mediante pruebas de Levene (Quinn \& Keough 2002). Cuando fue necesario, se realizaron transformaciones logarítmicas para optimizar normalidad y homogeneidad de variancias.

Se evaluó en qué grado cada especie cumple los criterios de especificidad y fidelidad a las condiciones de los sitios por medio del método del Valor Indicador (VI) (Dufrêne \& Legendre 1997). Se calcularon los valores indicadores para cada especie sobre la base de una matriz de abundancia de las especies, y se evaluó la significancia estadística de cada valor indicador mediante un procedimiento de aleatorización, considerando los factores fuego (no quemado y recientemente quemado) y ganado (ausencia y presencia).Una especie se determinó como característica de una condición si VI > 25\% y $P<0.05$ (Dufrêne \& Legendre 1997).

A partir de las tasas de ramoneo por especie y por parcela evaluadas a campo, se calculó para cada sitio el índice de ramoneo promedio, que indica la intensidad de ramoneo sobre todas las especies en el sitio muestreado (IRP; Veblen et al. 1992; Blackhall et al. 2008) y se calcula a partir de la suma estadística de todas las tasas de ramoneo (TR) para cada especie y para todas las especies registradas en cada sitio, en cada micro-parcela. Para los sitios con ganado, se realizó un análisis no paramétrico de Mann-Whitney para determinar si existían diferencias significativas entre los IRP de los sitios no quemados y recientemente quemados (Conover 1980). 


\section{Resultados \\ Condiciones micro-ambientales}

La temperatura media del aire en los sitios recientemente quemados difirió significativamente de la de los sitios no quemados, y este efecto varió según la hora del día (Tabla 2, Figura 1). Durante el día la temperatura fue significativamente mayor en los sitios recientemente quemados (en promedio entre $3.5^{\circ} \mathrm{C}$ y $2^{\circ} \mathrm{C}$, a las $12: 00$ y 16 : $00 \mathrm{~h}$ respectivamente). En cambio desde las 20:00h hasta las 8:00h, en los sitios quemados la temperatura fue significativamente menor a la de los no quemados (en promedio hasta $1.4{ }^{\circ} \mathrm{C}$ menos), sin que haya interacciones significativas. Además se registró durante la medianoche $(0: 00 \mathrm{~h})$ una disminución significativa de la temperatura en los sitios con ganadería en comparación a los sitios sin ganadería (en promedio $0.8^{\circ} \mathrm{C}$ ). La amplitud térmica promedio en la temporada fue significativamente mayor en los sitios quemados $\left(15.15 \pm 0.65(\mathrm{EE}){ }^{\circ} \mathrm{C}\right.$ y $11.21 \pm 0.61$

Tabla 2. Resultados obtenidos a partir de los ANOVAs factoriales $2 \times 2($ Fuego $=$ no quemado/recientemente quemado; Ganado= ausencia/presencia) para la temperatura media del aire $\left({ }^{\circ} \mathrm{C}\right)$ y la humedad relativa (\%), cada cuatro horas a lo largo de las 24 horas del día.* $P<0.05$ y ${ }^{* *} P<0.01$.

Table 2. Results of $2 \times 2$ ANOVAs (Fire= unburned/ recently burned; Cattle $=$ absence $/$ presence) for mean air temperature $\left({ }^{\circ} \mathrm{C}\right)$ and relative humidity $(\%)$, every four hours throughout 24 hour days. ${ }^{*} P<0.05 \mathrm{y}{ }^{* *} P<0.01$.

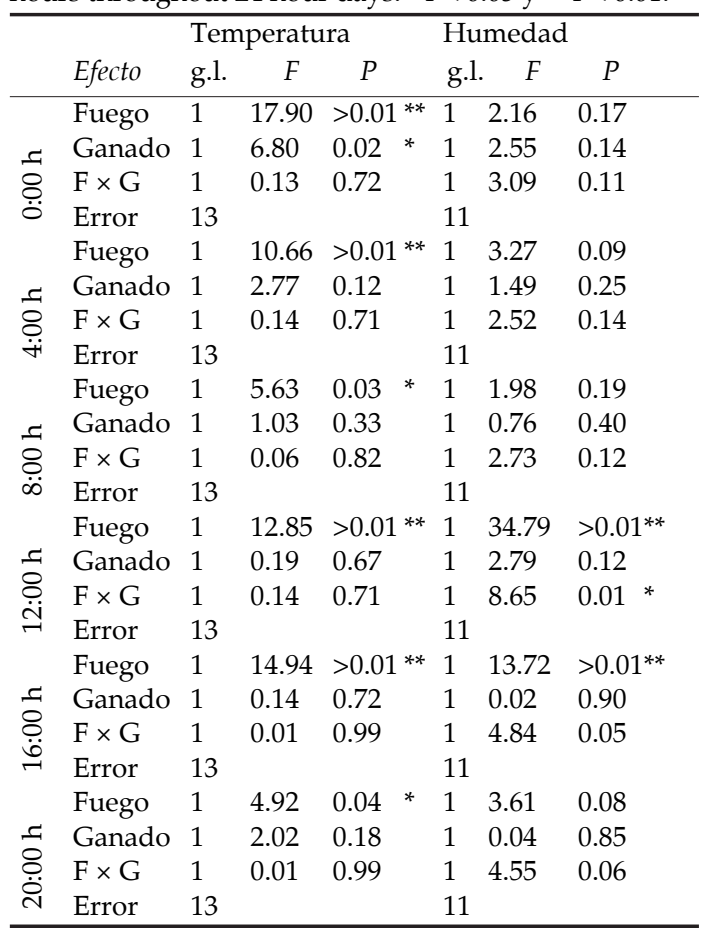

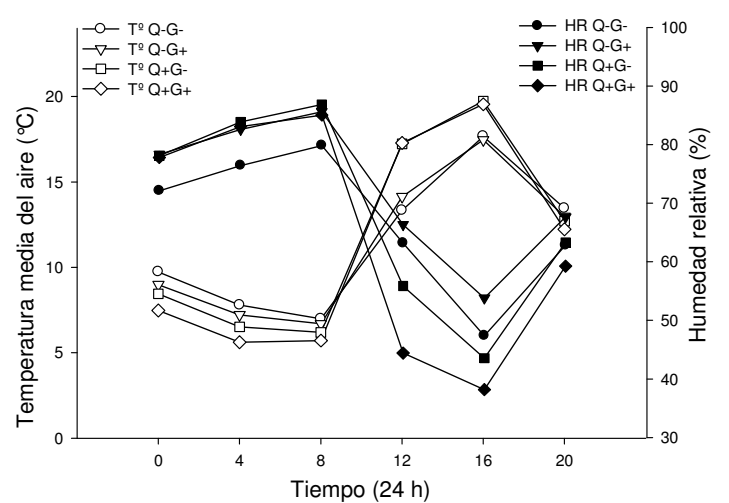

Figura 1. Temperatura media del aire $\left({ }^{\circ} \mathrm{C}\right)$ y humedad relativa (\%), medidas cada cuatro horas durante las 24 horas del día a $150 \mathrm{~cm}$ del suelo. Se muestran las mediciones diarias desde diciembre de 2009 hasta fines de marzo de 2010. Los valores son promedios para cada tipo de sitio: no quemados y sin ganado (Q- G-), no quemados y con ganado $(\mathrm{Q}-\mathrm{G}+)$, recientemente quemados y $\sin$ ganado (Q+ G-) y sitios recientemente quemados y con ganado $(\mathrm{Q}+\mathrm{G}+)$.

Figure 1. Mean air temperature $\left({ }^{\circ} \mathrm{C}\right)$ and relative humidity (\%), measured every four hours during 24 hour days, at $150 \mathrm{~cm}$ above ground. Daily measures from December 2009 to end of March 2010. Mean values for each type of site: unburned without cattle (Q- G-), unburned with cattle $(\mathrm{Q}-\mathrm{G}+)$, recently burned without cattle $(\mathrm{Q}+\mathrm{G}-)$ and recently burned with cattle $(\mathrm{Q}+\mathrm{G}+)$.

$\left(\right.$ EE) ${ }^{\circ} \mathrm{C}$ respectivamente; $F_{1,13}=17.03 ; P<0.01$ ), mientras que no se registraron diferencias entre sitios con y sin ganado $\left(F_{1,13}=0.17 ; P=\right.$ $0.07)$, ni una interacción significativa entre ambos disturbios $\left(F_{1,13}=0.05 ; P=0.82\right)$.

Desde las 20:00h hasta las 8:00h no se registraron efectos del fuego, el ganado o su interacción sobre la humedad relativa $(P>0.05$; Tabla 2, Figura 1). En las horas de mayor radiación solar (12:00h-16:00h) la humedad relativa fue en promedio un $80 \%$ menor en los sitios quemados en comparación a los sitios no quemados $(P<0.01)$. Si bien no se registró efecto principal del ganado sobre ambos horarios, sí se registró una interacción significativa entre el efecto del fuego y el ganado sobre la humedad relativa durante las horas del mediodía (12:00h), siendo menor la humedad en los sitios quemados y pastoreados que en los restantes sitios (Tukey, $P<0.05)$.

La proporción de radiación directa fue mayor en los sitios quemados en comparación a los sitios no quemados $\left(F_{1,13}=60.48, P<0.01\right.$; Figura 2a). En los sitios no quemados existe un dosel arbóreo que disminuyó la luz que llegó al sotobosque. El efecto del ganado, y su interacción con el fuego, no influyeron sobre la proporción de radiación directa $\left(F_{1,13}=1.84, P=\right.$ 0.19, y $F_{1,13}=4.25, P=0.06$, respectivamente). De 
a)

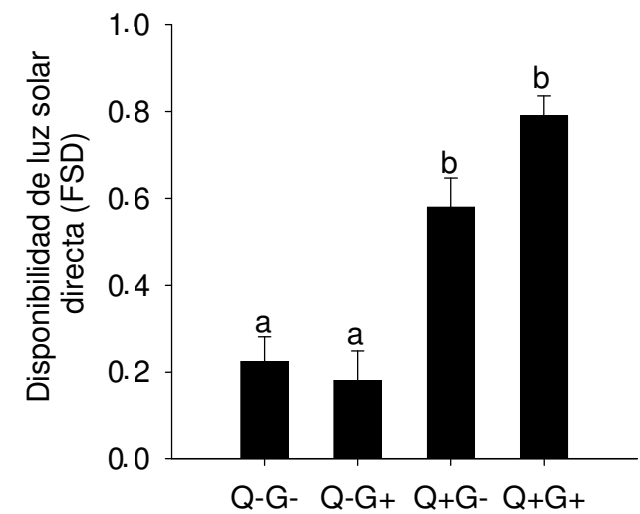

b)

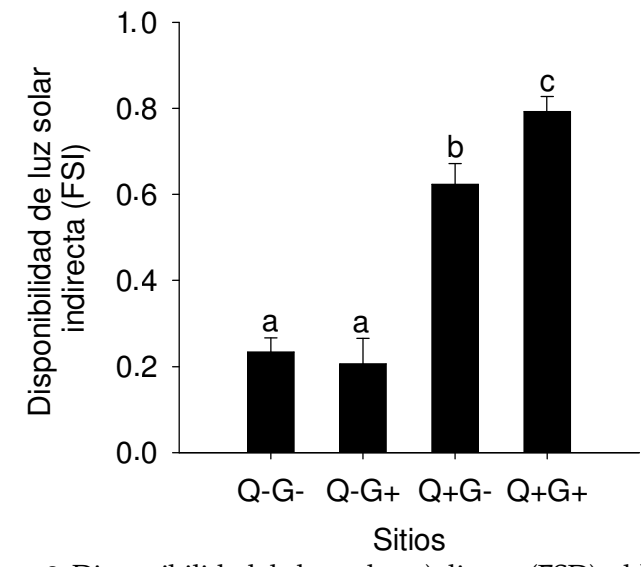

Figura 2. Disponibilidad de luz solar: a) directa (FSD) y b) indirecta (FSI). Los valores son promedios para cada tipo de sitio: no quemados y sin ganado (Q- G-), no quemados y con ganado (Q- G+), recientemente quemados y sin ganado $(\mathrm{Q}+\mathrm{G}-)$ y sitios recientemente quemados y con ganado $(\mathrm{Q}+\mathrm{G}+)$. Las barras indican el error estándar. Letras diferentes indican diferencias significativas entre sitios (Tukey, $P<0.05$ ).

Figure 2. Total radiation: a) direct site factor (FSD) and b) indirect site factor (FSI). Mean values for each type of site: unburned without cattle (Q- G-), unburned with cattle (Q$\mathrm{G}+$ ), recently burned without cattle (Q+ G-) and recently burned with cattle $(\mathrm{Q}+\mathrm{G}+)$. Bars indicate standard error. Different letters above bars indicate significant differences between sites (Tukey, $P<0.05$ ).

la misma manera, la proporción de radiación indirecta varió significativamente según el efecto del fuego, mientras que el ganado por sí sólo no influyó sobre esta variable $\left(F_{1,13}=115.01, P<0.01\right.$, y $F_{1,13}=2.41, P=0.14$, respectivamente; Figura $2 b$ ). La radiación indirecta varió además según el efecto combinado del fuego y el ganado, siendo mayor en los sitios recientemente quemados $\mathrm{y}$ con ganado (efecto fuego $\times$ ganado: $F_{1,13}=$ 4.67, $P=0.04$; Tukey, $P<0.05$; Figura $2 b$ ).

\section{Vegetación}

Las especies más abundantes y registradas en todos los sitios fueron C. culeou y $S$. patagonicus en primer y segundo lugar (Anexo I, Información suplementaria). Además se registraron con abundancias promedio $\geq 5 \%$ para todos los sitios: $V$. nigricans, $D$. juncea, N. antarctica, Berberis buxifolia y L. hirsuta. En promedio para todos los sitios estas siete especies componen el $71 \%$ de la cobertura vegetal. La cobertura total de especies leñosas y semi-leñosas fue en promedio mayor al $60 \%$ en todos los sitios. A su vez, la cobertura total promedio no varió según el efecto independiente del fuego y el ganado, pero sí lo hizo en respuesta al efecto combinado de ambos disturbios; la cobertura en los sitios quemados y con ganado fue menor que en los afectados por solo un disturbio (efecto fuego:

a)

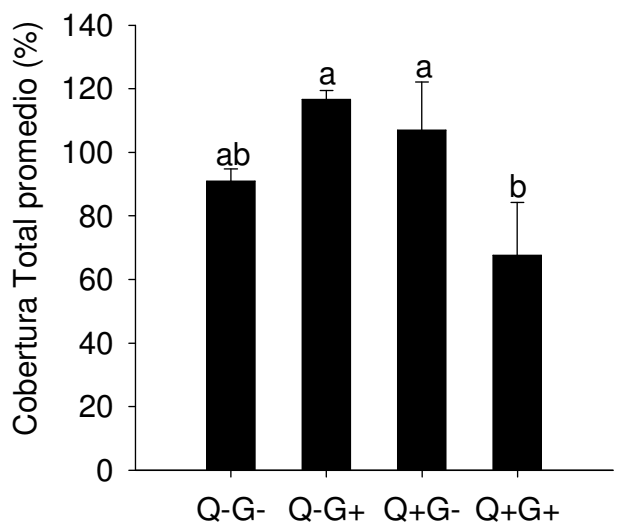

b)

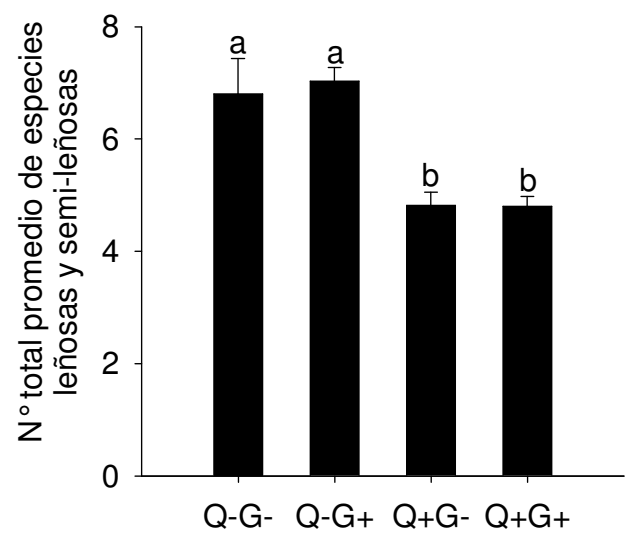

Figura 3. a) Cobertura total (\%), y b) número total de especies leñosas y semi-leñosas. Los valores son promedios para cada tipo de sitio: no quemados y sin ganado (Q- G-), no quemados y con ganado (Q- G+), recientemente quemados y $\sin$ ganado $(\mathrm{Q}+\mathrm{G}-)$ y sitios recientemente quemados y con ganado $(Q+G+)$. Las barras indican el error estándar. Letras diferentes indican diferencias significativas entre sitios (Tukey, $P<0.05$ ).

Figure 3. a) Total cover (\%), and b) total number of woody and semi-woody species. Mean values for each type of site: unburned without cattle (Q- G-), unburned with cattle (Q$\mathrm{G}+)$, recently burned without cattle $(\mathrm{Q}+\mathrm{G}-)$ and recently burned with cattle $(\mathrm{Q}+\mathrm{G}+)$. Bars indicate standard error. Different letters above bars indicate significant differences between sites (Tukey, $P<0.05$ ). 
$F_{1,13}=1.83, P=0.20$; efecto ganado: $F_{1,13}=0.31$, $P=0.59$; efecto fuego $\times$ ganado: $F_{1,13}=7.18, P=$ 0.02; Tukey, $P<0.05$; Figura 3a). La cobertura de especies leñosas y semi-leñosas exóticas no superó en ningún sitio más del 5\%, y la cobertura promedio total de las mismas para todos los sitios fue de $2.63 \%$. El número total promedio de especies fue significativamente menor en los sitios quemados en comparación con los sitios no quemados $\left(F_{1,13}=34.99, P<\right.$ 0.01 ; Figura $3 b)$. No se observaron diferencias significativas en el número de especies debido al efecto del ganado, ni a su interacción con el fuego $\left(F_{1,13}=0.08, P=0.78\right.$, y $F_{1,13}=0.12, P=$ 0.74 , respectivamente). La riqueza de especies exóticas fue baja, en promedio representó para todos los sitios el 3.84\% del total de especies registradas. La cobertura del dosel arbóreo fue similar entre los sitios no quemados con y $\sin$ ganado $(60.3 \pm 6.8 \%$ y $66.5 \pm 6.1 \%$ respectivamente; $F_{1,6}=0.46, P=0.52$ ).

Las especies indicadoras que caracterizaron los sitios no quemados al menos en los últimos 50 años, independientemente del efecto del ganado, fueron Maytenus chubutensis (VI= 97.54; $P<0.01)$ y la leñosa exótica Rosa rubiginosa $(\mathrm{VI}=88.24 ; P<0.01)$. También los sitios no quemados se caracterizaron por la presencia y abundancia en el sotobosque de renovales y juveniles de dos especies arbóreas muy frecuentes y dominantes en bosques maduros: $A$. chilensis (VI $=62.5 ; P<$ $0.01)$ y $N$. dombeyi $(\mathrm{VI}=50 ; P<0.01)$. Otras especies, que a pesar de no mostrar valores significativos para los sitios no quemados, mostraron valores muy altos de VI (VI> 40; $P>0.05)$ fueron Berberis darwinii, C. culeou, $L$. hirsuta, Gaultheria phillyreifolia, M. boaria, S. patagonicus y Embothrium coccineum. En los sitios quemados, cinco especies mostraron valores muy altos de VI ( $>40 ; P>0.05): R$. magellanicum, $V$. nigricans, B. buxifolia, $N$. antarctica y D. juncea.

Ribes magellanicum, especie muy palatable, fue la especie que mejor caracterizó los sitios sin ganado $(\mathrm{VI}=91.46 ; P<0.01)$. Otras especies que mostraron valores muy altos de VI aunque no mostraron valores significativos (VI> 40; $P>0.05$ ) en los sitios sin ganadería, fueron $V$. nigricans, $M$. boaria, S. patagonicus, L. hirsuta y Mutisia spinosa. La especie que mejor caracterizó los sitios con ganado fue la espinosa y poco palatable $B$. darwinii $(\mathrm{VI}=80.51 ; P<0.01)$. Además, cinco especies mostraron valores muy altos de VI (VI > 40; $P>$ 0.05): B. buxifolia, C. culeou, G. phillyreifolia, D. juncea y $N$. antarctica.

\section{Herbivoría}

En los sitios sin ganado no quemados y recientemente quemados no se registró herbivoría. El índice de ramoneo promedio fue similar y de alta intensidad en todos los sitios con ganado, tanto en los sitios no quemados como en los sitios recientemente quemados (IRP promedio de $4.59 \pm 0.38$ (ES), y $4.12 \pm 1.09$ (ES), respectivamente; test de Mann-Whitney: $\mathrm{U}=8, P>0.05)$. Estos valores registrados son similares a los encontrados en otros trabajos regionales realizados en sitios con impacto de ungulados exóticos con una mediana a alta presión de herbivoría (Relva \& Veblen 1998; Blackhall et al. 2008; Sasal 2009; Raffaele et al. 2011).

\section{DisCUSIÓN}

Los resultados de este trabajo muestran un efecto contundente del tiempo transcurrido desde el último incendio y en menor medida del ganado sobre las variables microambientales, las variables de la vegetación estudiadas, y especialmente sobre las especies indicadoras. Estos efectos, sólo en algunos casos sinérgicos y en ningún caso antagónico, pueden a futuro modificar las trayectorias de sucesión de las comunidades.

Las condiciones micro-ambientales fueron más extremas en los sitios recientemente quemados (i.e. ausencia de dosel arbóreo y en consecuencia mayor amplitud térmica, menor humedad relativa durante el día y mayor disponibilidad de luz solar), y en dos casos fueron aún más rigurosas en presencia del ganado vacuno (i.e. mayor disponibilidad de luz y menor humedad durante las horas de mayor radiación), produciendo ambos disturbios un efecto sinérgico sobre las condiciones adversas a nivel de micro-sitio. En estosambientes bajocondiciones más extremas, como ocurre en otros ambientes disturbados (Maestre et al. 2009), la disponibilidad de micro-sitios para el establecimiento de especies es brindada principalmente por especies nodrizas (Raffaele \& Veblen 1998). En estudios previos en la región se mostró que la radiación directa y la disponibilidad de agua en el suelo a nivel de micro-sitio, son factores claves en el establecimiento y supervivencia de plántulas de especies arbóreas de gran importancia en la región como A. chilensis y N. pumilio (Kitzberger et al. 2000, 2005). Un aumento y disminución respectivamente de estas condiciones micro-ambientales pondría en riesgo la regeneración de las especies 
nodrizas y consecuentemente el reclutamiento y establecimiento de estas especies arbóreas. Asimismo se ha registrado que la cobertura de especies, independientemente de su identidad, incide sobre los procesos de facilitación en el reclutamiento y supervivencia de $A$. chilensis (Kitzberger et al. 2000; Nuñez et al. 2009). En el presente trabajo se encontró que el fuego reciente y la herbivoría de ganado vacuno, actuando en combinación, disminuyeron la cobertura de especies leñosas y semi-leñosas reduciendo la disponibilidad de micro-sitios capaces de facilitar el establecimiento de especies. La reducción de la biomasa debida al fuego y al efecto directo e indirecto de los herbívoros (e.g. ramoneo, pisoteo, echaderos, cambios de nutrientes en el suelo, entre otros), puede cambiar las condiciones microambientales, como se registró en el presente trabajo (e.g. radiación y humedad microambiental).

Por otro lado, los cambios micro-ambientales pueden asociarse directamente a cambios en las características de las especies. En los sitios recientemente quemados, una mayor radiación, asociada a mayores temperaturas y menor humedad influyen directamente sobre las condiciones de los combustibles presentes en un ambiente y por ende sobre las probabilidades de ignición y propagación de un incendio (Bond \& van Wilgen 1996; Blackhall et al. 2012). Mientras mayor sea la tasa de desecación y menor la humedad de la vegetación (combustible), menor será la cantidad de energía necesaria para desencadenar una ignición (Rundel 1981). Blackhall et al. (2012) determinaron para cuatro de las siete especies más frecuentes encontradas en este estudio, S. patagonicus, D. juncea, N. antarctica, y L. hirsuta, que la humedad foliar de las plantas de estas especies creciendo en sitios recientemente quemados era menor a la de las plantas creciendo en sotobosques no quemados.

El análisis de especies indicadoras permitió registrar el efecto del fuego y la herbivoría sobre las especies que caracterizan una comunidad. Dos especies caracterizaron los sitios no quemados al menos en los últimos 50 años: $M$. chubutensis (arbusto típico de condiciones húmedas y sombrías; San Martín et al. 1991) y R. rubiginosa (arbusto exótico e invasor de bosques y matorrales andinopatagónicos; Damascos \& Gallopin 1992). En asociación a la herbivoría, la especie que caracterizó los sitios sin presencia de ganado fue R. magellanicum. Algunos de los numerosos efectos del ganado documentados sobre esta especie muy palatable son: 1) reducción de la producción de flores y frutos, y viabilidad de las semillas de las plantas creciendo bajo herbivoría intensa (de Paz \& Raffaele 2013); 2) aumento de la proporción de combustible altamente inflamable (i.e. combustible fino) y biomasa por unidad de volumen en plantas ramoneadas por ganado (Blackhall et al. 2015); y 3) en sitios sobre-pastoreados, presencia únicamente en refugios naturales de difícil acceso (e.g. entre troncos caídos; Blackhall et al. 2008). En los sitios con ganado la especie indicadora fue $B$. darwinii, poco palatable y espinosa, y abundante en sitios disturbados (Raffaele et al. 2011). Esta especie, junto con otras de baja palatabilidad, se ven favorecidas fenológicamente por la presencia de ganado vacuno, y este efecto se observa en la extensión de sus períodos de floración y una mayor producción de flores y frutos (de Paz \& Raffaele 2013). Las especies indicadoras encontradas en este estudio corroboran no sólo el efecto negativo del ganado sobre las especies palatables sino también el efecto indirecto positivo sobre especies de menor palatabilidad, ya registrados en trabajos anteriores considerando otras variables (Sasal 2009; Blackhall et al. 2008; Raffaele et al. 2011; de Paz \& Raffaele 2013).

En resumen, a través de diferentes mecanismos, el fuego y la presencia de ganado vacuno modifican las características de la comunidad vegetal, ya sea mediante cambios en las condiciones micro-ambientales, en la cobertura, en el número de especies, y/o en las especies que caracterizan el ambiente. Sólo en algunos casos se pudo determinar que estos cambios pueden ser mediados por el efecto combinado de ambos disturbios, como se observó para la humedad relativa, la disponibilidad de luz y la cobertura de especies leñosas y semileñosas. El conocimiento e identificación de las especies indicadoras y la caracterización del micro-sitio en donde estas especies crecen, brindan información básica para colaborar en el desarrollo de pautas de restauración, conservación de la biodiversidad, y manejo sustentable de los bosques y matorrales del noroeste patagónico.

\footnotetext{
Agradecimientos: Este trabajo fue financiado por la Universidad Nacional del Comahue (UNC B103 y UNC B152), UNC-PICT (Subsidio 01-07320), PICT 2012-2371 y subsidios No. 0117366, 0956552 y 0966472 de NSF, Estados Unidos. Agradecemos a AM Cingolani y a dos revisores anónimos por sus valiosos comentarios y sugerencias. Agradecemos a S Polcowñuk y E Milani por la asistencia en trabajo de campo. Agradecemos a la Administración
} 
de Parques Nacionales por los permisos otorgados para realizar este trabajo dentro del PNNH.

\section{BIBLIOGRAFÍA}

Administración de Parques Nacionales. 1999. Informe sobre las consecuencias ecológicas de los incendios forestales - Temporada 1998-1999. Parque Nacional Nahuel Huapi. Bariloche, Argentina.

Alauzis, MV; MJ Mazzarino; E Raffaele \& L Roselli. 2004. Wildfires in NW Patagonia: long-term effects on Nothofagus forest soil. Forest Ecol. Manag., 192:131-142.

Barros, V; V Cordon; C Moyano; R Mendez; J ForQuera \& O Pizzio. 1983. Cartas de precipitación de la Zona Oeste de las Provincias de Río Negro y Neuquén, Primera Contribución. Universidad Nacional del Comahue, Cinco Saltos, Argentina.

Blackhall, M. 2012. Respuestas de especies leñosas a herbívoros e incendios en bosques y matorrales del noroeste de la Patagonia: estudio de la inflamabilidad vegetal. Tesis Doctoral, Universidad Nacional del Comahue. Bariloche, Argentina

Blackhall, M; E RafFaele \& TT Veblen. 2008. Cattle affect early post-fire regeneration in a Nothofagus dombeyiAustrocedrus chilensis mixed forest in northern Patagonia, Argentina. Biol. Conserv., 141:2251-2261.

Blackhall, M; E Raffaele \& TT Veblen. 2012. Is foliar flammability of woody species related to time since fire and herbivory in northwest Patagonia, Argentina? J. Veg. Sci., 23:931-941.

Blackhall, M; TT Veblen \& E Raffaele. 2015. Recent fire and cattle herbivory enhance plant-level fuel flammability in shrublands. J. Veg. Sci., 26:123-133.

BOND, WJ. 2005. Large parts of the world are brown or black: a different view on the 'Green World' hypothesis. J. Veg. Sci., 16:261-266.

Bond, WJ \& BW van Wilgen. 1996. Fire and plants. Chapman \& Hall.

ColLINS, SL. 1987. Interaction of disturbances in tall-grass prairie: a field experiment. Ecology, 68:1243-1250.

CONOver, WJ. 1980. Practical non-parametric statistics. John Wiley \& Sons.

Correa, MN. 1969-1997. Flora Patagónica (República Argentina). Colección Científica del INTA.

Damascos, MA \& GG Gallopin. 1992. Ecología de un arbusto introducido (Rosa rubiginosa L. = Rosa eglanteria L.): riesgo de invasión y efectos en las comunidades vegetales de la región andino-patagónica de Argentina. Rev. Chil. Hist. Nat., 65:395-407.

DARLING, ES \& IM CÔTÉ. 2008. Quantifying the evidence for ecological synergies. Ecol. Lett., 11:1278-1286.

Donoso, C; L Steinke \& A Premoli. 2006. Nothofagus antarctica (G. Forster) Oerst. Pp. 401-410 en: DonOso ZEGERS C (ed.). Las especies arbóreas de los bosques templados de Chile y Argentina. Autoecología. Marisa Cuneo Ediciones.

DufrêNE, M \& P LEGENDRE. 1997. Species assemblages and indicator species: the need for a flexible asymmetrical approach. Ecol. Monograph., 67:345-366.

FuHLENDORF, SD; DM ENGLE; JAY KeRbY \& R HAMilton. 2009. Pyric herbivory: rewilding landscapes through the recoupling of fire and grazing. Conserv. Biol., 23: 588-598.

Hijmans, RJ; SE CAMERON; PG PARRA \& A JARVIS. 2005. Very high resolution interpolated climate surfaces for global land areas. Int. J. Climatol., 25:1965-1978.
KIRKPATRICK, JB; JB MARSDEN-SMEDLEY \& SWJ LEONARD. 2011. Influence of grazing and vegetation type on post"fire flammability. J. Appl. Ecol., 48:642-649.

Kitzberger, T; E Raffaele; K Heinemann \& MJ Mazzarino. 2005. Effects of fire severity in a North Patagonian subalpine forest. J. Veg. Sci., 16:5-12.

Kitzberger, T; DF Steinaker \& TT Veblen. 2000. Effects of climatic variability on facilitation of tree establishment in northern Patagonia. Ecology, 81:1914-1924.

Kitzberger, T \& TT Veblen. 1999. Fire-induced changes in northern Patagonian landscapes. Landscape Ecol., 14: $1-15$.

LAUría Sorge, RM \& CA Romero. 1999. La ganadería doméstica de los pobladores con permiso de ocupación y pastaje en tierras fiscales del Parque Nacional Nahuel Huapi. Informe, Administración de Parques Nacionales, Parque Nacional Nahuel Huapi, Argentina.

Maestre, FT; RM Callaway; F Valladares \& CJ LorTIE. 2009. Refining the stress-gradient hypothesis for competition and facilitation in plant communities. $J$. Ecol., 97:199-205.

Merino, ML; BN CARPInetTI \& AM AbBA. 2009. Invasive mammals in the National Parks system of Argentina. Nat. Areas J., 29:42-49.

Mermoz, M; T Kitzberger \& TT Veblen. 2005. Landscape influences on occurrence and spread of wildfires in patagonian forest and shrublands. Ecology, 86:27052715.

Mermoz, M \& CE Martín. 2005. Mapa de vegetación del Parque y la Reserva Nacional Nahuel Huapi. Anales de Parques Nacionales, 17:51-62.

Nuñez, CI; E Raffaele; MA Nuñez \& F CuAssolo. 2009. When do nurse plants stop nursing? Temporal changes in water stress levels in Austrocedrus chilensis growing within and outside shrubs. J. Veg. Sci., 20:1064-1071.

Paine, RT; MJ Tegner \& EA Johnson. 1998. Compounded perturbations yield ecological surprises. Ecosystems, 1: 535-545.

Paritsis, J; E Raffaele \& TT Veblen. 2006. Vegetation disturbance by fire affects plant reproductive phenology in a shrubland community in northwestern Patagonia, Argentina. New Zeal. J. Ecol., 30:387-395.

de Paz, M \& E Raffaele. 2013. Cattle change plant reproductive phenology, promoting community changes in a post-fire Nothofagus forest in northern Patagonia, Argentina. J. Plant Ecol., 6:459-467.

QuinN, GP \& MJ KeOUGH. 2002. Experimental design and data analysis for biologists. Cambridge University Press.

RAFFAELE, E \& TT VEBLEN. 1998. Facilitation by nurse shrubs of resprouting behaviour in a post-fire shrubland in northern Patagonia, Argentina. J. Veg. Sci., 9:693-698

Raffaele, E; TT Veblen; M Blackhall \& N TerceroBuCARDO. 2011. Synergistic influences of introduced herbivores and fire on vegetation change in northern Patagonia, Argentina. J. Veg. Sci., 22:59-71.

ReLVA, MA \& TT VEBLEN. 1998. Impacts of introduced large herbivores on Austrocedrus chilensis forests in northern Patagonia, Argentina. Forest Ecol. Manag., 108:27-40.

RICH, PM. 1990. Characterizing plant canopies with hemispherical photographs. Rem. Sens. Rev., 5:13-29.

Rundel, PW. 1981. Structural and chemical components of flammability. En: Fire regimes and ecosystem properties. USDA Forest Service General Tech. Rep. GTR-WO-26, Pp 183-207. Washington DC, USA.

San Martín, J; A Troncoso; A Mesa; T Bravo \& C Ramírez. 1991. Estudio fitosociológico del bosque caducifolio 
magallánico en el límite Norte de su área de distribución. Bosque, 12:29-34.

SASAL, Y. 2009. Efecto del Ganado en áreas incendiadas sobre la comunidad de artrópodos terrestres y las interacciones planta-insecto en el NO de la Patagonia, Argentina. Tesis Doctoral, Universidad Nacional del Comahue. Bariloche, Argentina.

Tercero-Bucardo, N; T Kitzberger; TT Veblen \& E Raffaele. 2007. A field experiment on climatic and herbivore impacts on post-fire tree regeneration in north-western Patagonia. J. Ecol., 95:771-779.

TuRNER, MG. 2010. Disturbance and landscape dynamics in a changing world. Ecology, 91:2833-2849.
Veblen, TT; A Holz; J Paritsis; E Raffaele; T Kitzberger \& M BlACKHALL. 2011. Adapting to global environmental change in Patagonia: What role for disturbance ecology? Austral Ecol., 36:891-903.

Veblen, TT; T Kitzberger; E Raffaele \& DC LoRenz. 2003. Fire History and Vegetation Changes in Northern Patagonia, Argentina. Pp. 265-293 en: TT Veblen; WL BAKer; G Montenegro \& TW Swetnam (eds.). Fire and Climatic Change in Temperate Ecosystems of the Western Americas. Springer-Verlag.

Veblen, TT; M Mermoz; C Martin \& T Kitzberger. 1992. Ecological impacts of introduced animals in Nahuel Huapi National Park, Argentina. Conserv. Biol., 6:71-83. 


\section{INFORMACIÓN SUPLEMENTARIA}

Anexo 1. Porcentajes promedio de cobertura de cada especie leñosa y semi-leñosa registrada en los sitios no quemados y sin ganado (Q- G-), no quemados y con ganado $(\mathrm{Q}-\mathrm{G}+)$, recientemente quemados y sin ganado $(\mathrm{Q}+\mathrm{G}-)$ y sitios recientemente quemados y con ganado $(\mathrm{Q}+\mathrm{G}+)$. Las especies están ordenadas para cada tipo de sitio según valores decrecientes de cobertura promedio. El símbolo $\boldsymbol{\omega}$ indica las especies exóticas.

Appendix 1. Mean cover percentages for each woody and semi-woody species at unburned without cattle (Q- G-), unburned with cattle $(\mathrm{Q}-\mathrm{G}+)$, recently burned without cattle $(\mathrm{Q}+\mathrm{G}-)$ and recently burned with cattle $(\mathrm{Q}+\mathrm{G}+)$ sites. For each type of site species are arranged according to decreasing values of mean cover. Symbol $\boldsymbol{\omega}$ indicate exotic species.

\begin{tabular}{|c|c|c|c|c|c|c|c|}
\hline Especies en Q- G- & & Especies en Q- G+ & & Especies en Q+ G- & & Especies en Q+ G+ & \\
\hline Chusquea culeou & 22.75 & Chusquea culeou & 40.25 & Vicia nigricans & 21.50 & Diostea juncea & 15.75 \\
\hline Schinus patagonicus & 16.02 & Schinus patagonicus & 14.13 & Chusquea culeou & 17.90 & Berberis buxifolia & 12.27 \\
\hline Aristotelia chilensis & 6.26 & Berberis darwinii & 12.39 & Nothofagus antarctica & 12.90 & Schinus patagonicus & 11.64 \\
\hline Lomatia hirsuta & 6.01 & Berberis buxifolia & 6.76 & Schinus patagonicus & 12.61 & Nothofagus antarctica & 8.76 \\
\hline Austrocedrus chilensis & 5.51 & Lomatia hirsuta & 6.63 & Diostea juncea & 9.40 & Chusquea culeou & 8.64 \\
\hline Maytenus chubutensis & 4.03 & Vicia nigricans & 6.26 & Lomatia hirsuta & 6.00 & Vicia nigricans & 4.89 \\
\hline Maytenus boaria & 3.40 & Nothofagus antarctica & 6.00 & Discaria chacaye & 4.70 & Mutisia spinosa & 3.00 \\
\hline Rosa rubiginosa $\boldsymbol{\varphi}$ & 3.38 & Maytenus chubutensis & 5.39 & Berberis buxifolia & 4.42 & Lomatia hirsuta & 1.00 \\
\hline Nothofagus antarctica & 3.25 & Rosa rubiginosa $\boldsymbol{\omega}$ & 5.09 & Ribes magellanicum & 4.21 & Berberis darwinii & 0.64 \\
\hline Diostea juncea & 2.75 & Gaultheria phillyreifolia & 4.63 & Mutisia spinosa & 3.81 & Gaultheria phillyreifolia & 0.63 \\
\hline Nothofagus dombeyi & 2.75 & Diostea juncea & 3.50 & Maytenus boaria & 2.00 & Buddleja globosa & 0.63 \\
\hline Mutisia spinosa & 2.50 & Austrocedrus chilensis & 1.63 & Fabiana imbricata & 1.60 & Maytenus boaria & 0.26 \\
\hline Vicia nigricans & 2.01 & Maytenus boaria & 1.16 & Baccharis magellanica & 1.20 & Ribes cucullatum & 0.26 \\
\hline Ribes magellanicum & 1.52 & Embothrium coccineum & 0.63 & Rosa rubiginosa $\boldsymbol{\omega}$ & 1.01 & Ribes magellanicum & 0.16 \\
\hline Colletia hystrix & 1.37 & Berberis linearifolia & 0.63 & Berberis darwinii & 1.00 & Maytenus chubutensis & 0.13 \\
\hline Gaultheria phillyreifolia & 1.25 & Ribes magellanicum & 0.41 & Nothofagus pumilio & 1.00 & Juniperus communis $\boldsymbol{\varphi}$ & 0.01 \\
\hline Juniperus communis $\boldsymbol{\emptyset}$ & 1.01 & Crataegus monogyna $\boldsymbol{\omega}$ & 0.38 & Mulinum spinosum & 0.60 & Mutisia decurrens & 0.01 \\
\hline Berberis buxifolia & 0.89 & Mutisia spinosa & 0.38 & Embothrium coccineum & 0.40 & Berberis serrato-dentata & 0.01 \\
\hline Myoschilus oblongum & 0.88 & Nothofagus dombeyi & 0.25 & Gaultheria phillyreifolia & 0.30 & Nothofagus pumilio & 0.01 \\
\hline Baccharis obovata & 0.50 & Ribes cucullatum & 0.13 & Maytenus chubutensis & 0.11 & Baccharis obovata & 0.01 \\
\hline Gaultheria mucronata & 0.50 & Mutisia decurrens & 0.13 & Baccharis obovata & 0.10 & & \\
\hline Mutisia decurrens & 0.38 & Myoschilus oblongum & 0.01 & Mutisia decurrens & 0.10 & & \\
\hline Discaria chacaye & 0.38 & Lathyrus magellanicum & 0.01 & Lathyrus magellanicum & 0.10 & & \\
\hline Gaultheria poeppigi & 0.38 & & & Ribes cucullatum & 0.01 & & \\
\hline Escallonia rubra & 0.37 & & & Berberis serrato-dentata & 0.01 & & \\
\hline Embothrium coccineum & 0.25 & & & & & & \\
\hline Cynanchum descolei & 0.25 & & & & & & \\
\hline Ribes cucullatum & 0.13 & & & & & & \\
\hline Cytisus scoparius $\boldsymbol{(}$ & 0.13 & & & & & & \\
\hline Berberis darwinii & 0.02 & & & & & & \\
\hline
\end{tabular}

